\title{
CONSERVATION RISK ASSESSMENT: A STRATEGY FOR MANAGING RESOURCES FOR PREVENTIVE CONSERVATION
}

\section{Robert Waller}

\section{ABSTRACT}

Ideally, practitioners of preventive conservation should be able to quantify all risks to a collection and determine the most cost-effective means of reducing overall risk to that collection. This paper describes an attempt by the Canadian Museum of Nature to assess the risks to collections. Currently. the information required to produce accurate estimates of the magnitude of many risks is lacking. Nevertheless, simply attempting the exercise among a group of collections care staff produces several valuable results. Staff are made aware of the range of risks affecting collections. For certain risks, low-cost or no-cost methods of reducing the risk can be identified immediately. Conservation can use information about the uncertainties of estimates to develop improved methods for documenting damage and can develop research plans that address priority issues. Finally, the information obtained, while limited in accuracy, is coherent and comparable between diverse collections. This enables managers to make informed decisions on allocating resources for preventive conservation.

\section{INTRODUCTION}

Ideally, preventive conservation should be able to identify and quantify all risks to a collection or set of collections. We do not currently have the knowledge required to do this precisely. Nevertheless, by attempting to quantify all risks to collections, useful estimates can be obtained of the relative magnitudes of most risks. This paper briefly describes the method used to accomplish this at the Canadian Museum of Nature, Ottawa. Results for three collections are briefly presented. The cost, benefits and difficulties associated with this exercise are discussed.

This paper does not pretend to present an optimal method of assessing risks. Its purpose is to demonstrate that, despite current limitations of knowledge, it is possible to obtain useful estimates of the relative magnitudes of many risks. Further, there are numerous benefits to be derived simply from working through the exercise of a risk assessment.

\section{METHOD}

2.1 Identifying risks

\subsubsection{Agents of deterioration}

Ten agents of deterioration are recognized. These include the nine identified by Michalski: physical forces; criminals; fire; water; pests; contaminants; light, UV; incorrect temperature; and incorrect relative humidity [1]. The tenth agent is termed custodial neglect. It includes loss of specimens through misplacement, failure to obtain legal title for acquisitions, failure permanently to record specimen data and effectively to link them to specimens, and a range of other intellectual and legal shortcomings that reduce the utility and value of collections.

A comprehensive system for grouping risks in categories is essential to develop a useful risk assessment methodology. It is also essential that staffs involved in performing the risk assessments are convinced that the system is comprehensive. This was accomplished by having staff work in small groups to identify as many diverse risks to collections as possible. Then, working as a group. they were able to fit all identified risks into the framework of the 10 agents of deterioration.

\subsubsection{Types of risk}

The concept of risk management is appearing ever more frequently in the conservation literature [2, 3]. However, authors generally concentrate on elimination or mitigation of risks due to disasters. Assessment of total risk to collections requires that less

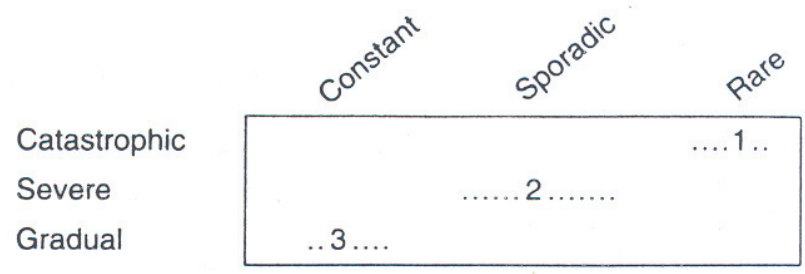

Fig. 1 Three types of risk are identified according to their frequency and severity.

catastrophic risks also be considered $[4,5]$.

To facilitate this, three types of risk are recognized. These range from 'type 1 " defined as rare and catastrophic, to 'type 3', which are constant and gradual. Between these extremes are 'type 2' risks, which are sporadic and intermediate in severity. The relation between these somewhat arbitrarily defined types of risk is shown schematically in Figure 1. By way of example, within the group of risks related to the agent 'physical forces', an event such as an earthquake would be considered a type 1 risk; an event such as dropping a drawerful of specimens while rearranging drawers within a cabinet would be considered a type 2

Table 1 Specific examples of types of risk identified for each agent of deterioration.

\begin{tabular}{|c|c|}
\hline $\begin{array}{l}\text { Categories of risk } \\
\text { (agent - type) }\end{array}$ & Examples \\
\hline Physical forces - Type 1 & $\begin{array}{l}\text { Earthquake, building structure collapse, } \\
\text { etc. }\end{array}$ \\
\hline Physical forces - Type 2 & $\begin{array}{l}\text { Dropping specimens, damage while plac- } \\
\text { ing specimens, etc. }\end{array}$ \\
\hline Physical forces - Type 3 & $\begin{array}{l}\text { Distortion from poor support, constant } \\
\text { vibration, etc. }\end{array}$ \\
\hline Fire & Fire \\
\hline Water-Type 1 & Flood \\
\hline Water - Type 2 & Roof leaks, plumbing leaks, etc. \\
\hline Water - Type 3 & Rising damp \\
\hline Criminals - Type I & Major theft \\
\hline Criminals - Type 2 & Isolated instances of theft or vandalism \\
\hline Criminals - Type 3 & $\begin{array}{l}\text { Embezzlement by staff or frequent users of } \\
\text { the collection }\end{array}$ \\
\hline Pests & Pest infestation \\
\hline Contaminants - Type 1 & $\begin{array}{l}\text { Fallout from nearby industrial or transport } \\
\text { accident, etc. }\end{array}$ \\
\hline Contaminants - Type 2 & $\begin{array}{l}\text { Use of a corrosive cleaner, dust during } \\
\text { construction, etc. }\end{array}$ \\
\hline Contaminants - Type 3 & $\begin{array}{l}\text { Gases and vapours from storage hardware, } \\
\text { acidity in fluids, etc. }\end{array}$ \\
\hline Light and UV radiation & Fading of colours, structural damage, etc. \\
\hline Incorrect temperature - Type 1 & Thawing of a frozen tissue collection \\
\hline Incorrect temperature - Type 2 & $\begin{array}{l}\text { Thermal shock to susceptible specimens, } \\
\text { etc. }\end{array}$ \\
\hline Incorrect temperature - Type 3 & Higher than ideal \\
\hline $\begin{array}{l}\text { Incorrect relative humidity - } \\
\text { Type } 2\end{array}$ & $\begin{array}{l}\text { High enough to cause an outbreak of } \\
\text { mould or mildew, rapid change in concen- } \\
\text { tration of fluids causing damage from } \\
\text { osmotic pressure, etc. }\end{array}$ \\
\hline $\begin{array}{l}\text { Incorrect relative humidity - } \\
\text { Type } 3\end{array}$ & $\begin{array}{l}\text { Splitting shells or teeth, transitions in } \\
\text { minerals, deterioration of fluid preserves, } \\
\text { etc. }\end{array}$ \\
\hline Custodial neglect - Type I & Collection abandonment \\
\hline Custodial neglect - Type 2 & Loss of specimens, specimen data, etc. \\
\hline Custodial neglect - Type 3 & $\begin{array}{l}\text { Ongoing failure to ensure ownership, easy } \\
\text { access, etc. }\end{array}$ \\
\hline
\end{tabular}

This compilation is intended as illustrative rather than comprehensive. Seperate examples are given for fluid-preserved collections where these differ significantly from the nature of the equivalent risk to dry collections. 
risk; and the distortion of an improperly supported specimen due to creep would be considered a type 3 risk. Risks due to certain of the agents of deterioration occur as only one of the types. Fire would always be considered a type 1 risk and pests would generally be considered a type 2 risk. Table 1 provides examples of each type of risk that was considered for each agent of deterioration in the assessment exercise. Frequently, a collection will be threatened by more than one risk of a given type for a given agent of deterioration. The term 'category of risk' is used to group risks of a common type for a given agent of deterioration.

Types of risk are defined, not only to help ensure that risks are considered comprehensively, but also to clarify that different strategies and sources of information are required to estimate the magnitude of the different types of risk. The estimation of the magnitude of type 1 risks is highly dependent on statistical information regarding the probability of events. This is not an area in which most conservators have expertise and, therefore, help must be obtained from experts in areas such as security and fire control. The estimation of type 2 risks requires the kind of information that is primarily provided by condition reports associated with use of collections, for exhibit or research. Estimation of type 3 risks is ultimately best based on the results of conservation research combined with a knowledge of the environmental conditions affecting a collection. At present, we lack the specific information required for accurate estimation of most risks. It is usually possible, however, to obtain reasonable, order of magnitude, estimates based on the experience of staff and colleagues in collection care.

\subsection{Estimating magnitudes of risk}

In business, there are many methods for estimating risk and some excellent texts are now available $[6,7]$. For the assessment of risks to collections, three out of four possible quantities were considered, depending on the type of risk. These quantities included either 'probability' or 'extent', and both 'fraction susceptible' and 'loss in value'.

Probability. (P) is the estimated chance of occurrence of an event of a given severity. For this assessment, risk was considered over a projected 100 -year period. In reality, there will be a range of probabilities associated with a range of severities for any given type 1 risk. Consider the risk of damage due to earthquakes. The most significant threat to our collections from earthquakes was found to be from the displacement of unrestrained objects on shelves or in drawers. The average distance travelled by an unrestrained object is one useful way to characterize the severity of an earthquake. The probability that in a 100 year period an earthquake will occur which causes unrestrained objects to move by one millimeter is very high, essentially one. The probabilities associated with stronger earthquakes which would cause larger displacements would become progressively less, approaching zero for very large displacements. This is shown schematically in Figure 2.

It would be extremely difficult for a conservator or a collection manager to consider this range of probabilities and severities, assuming that they are known, and from that estimate the risk to a collection. The strategy adopted for overcoming this difficulty was to choose a magnitude of displacement for which the probability of that event occurring over a 100 year period would be approximately $50 \%$ (Fig. 2). Collection managers were then asked to consider the effect on their collection of forces that would cause an average displacement of about $10 \mathrm{~cm}$. Similar simple models were used to characterize the probability of other type 1 risks. Wherever possible, outside experts are consulted for verification of the validity of the simple model chosen.

Extent (E) is used in place of probability for all type 3 and most type 2 risks. In these instances, the probability of an occurrence is

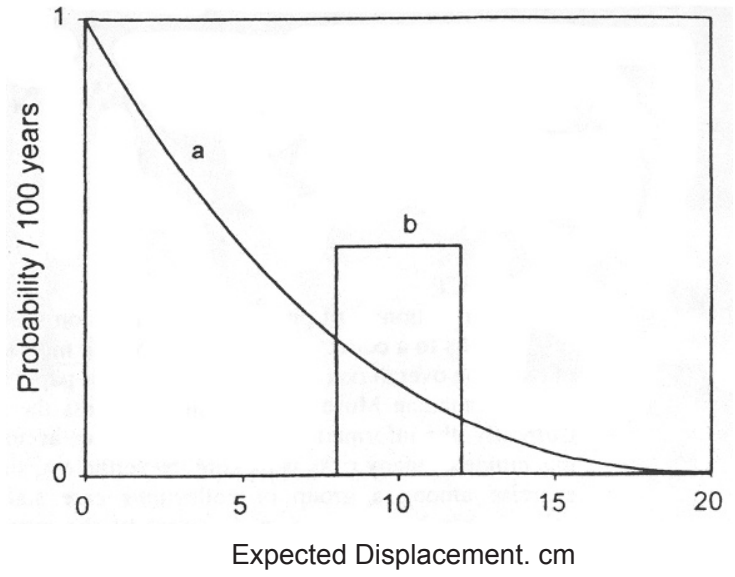

Fig. 2 (a) Schematic representation of the distribution of probability for earthquakes which would cause a given expected displacement of an unrestrained object. (b) The simple model used to estimate the significance of the risk due to earthquakes in terms of the average displacement of unrestrained objects.

high, usually one, and it is the extent to which the process occurs that is, or can be, limited. As an example, consider that all dried, pressed plant specimens are subject to physical damage during handling. Over a $100-$ year period, virtually all specimens in the collection will be handled and many will suffer some physical damage, with consequent loss in value. The extent to which damage will occur is the factor that can be estimated, based on the experience of collection managers, and is most useful for defining the magnitude of the risk.

Fraction susceptible (FS) defines that part of a collection which is considered vulnerable to a particular risk. In some instances, this will comprise a well-defined subset of the collection. For example, within the mineral collection, a predictable set of hydrated species is subject to damage (efflorescence) through exposure to an incorrect relative humidity. In other cases, susceptibility is a continuously variable characteristic. In these cases, a simplifying model, similar to that used for probability of a disaster, is used (Fig. 3). For example, the susceptibility of mineral specimens to professional thieves is highest for precious metals and gemstones, lowest for bulky specimens with little monetary value, and varies continuously in between. To facilitate estimation of the risk of a major theft, the collection was considered to have a fraction susceptible and a fraction that was not susceptible. This provides a rough but workable model.

Loss in value (LV) is defined as the reduction in the value of a

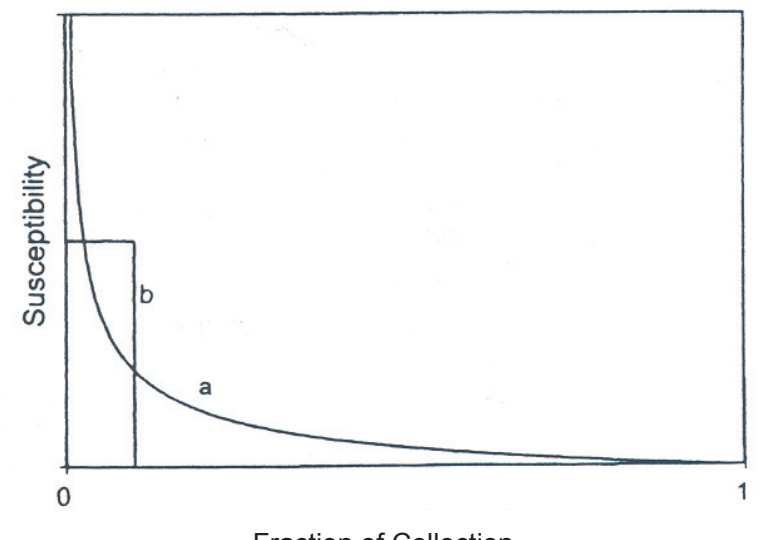

Fraction of Collection

Fig. 3 (a) The susceptibility of specimens or objects within a collection may be continuously variable. (b) A simple model is used to distinguish between a part of the collection that is considered to be highly susceptible and a part that is considered to have low or negligible susceptibility. 
collection for its intended purposes. Natural science collections are used primarily as research tools and the value considered here does not usually have a direct relation to a monetary or market value. An exception would be exhibit-quality mineral or gem specimens, for which market value can be a reasonable indication of display value. For the most part, however, the value considered here is related to the utility of the collection. Loss in value is one of the most difficult factors to estimate. It is very dependent on the way collections are used, and on which aspects of specimens contribute to their usefulness.

Magnitude of risk is taken to be the product of P x FS x LV for all type 1, and some type 2, risks and the product of E x FS x LV for other types of risk. For certain risk categories, the magnitude of risk will be the sum of two or more individual risks. In all examples encountered, the total magnitude for a risk could be taken as the simple sum of the component risks. In most instances, the use of the proper combinatorial calculations, rather than simple multiplication and summation, changes the results by an amount that is insignificant compared to the uncertainty of the estimated values used.

\section{RESULTS}

3.1 General

The Canadian Museum of Nature cares for approximately eight million specimens and objects. Collection care staff number approximately 30 .

In all, 19 risk assessments were completed to cover 15 collections housed in seven buildings. Some collections required more than one assessment, if subsets of the collection differed significantly in nature or were stored in significantly different facilities. Further, the data obtained from each assessment lose meaning when they are not considered together with the text describing the basis for estimating each factor. The data and the supporting documentation comprise several hundred pages of text and resist summary in this short paper. Consequently, only interim results, without their full rationale, are presented for three collections. These results will be used to demonstrate the manner in which risks were estimated and some of the problems and benefits of this risk assessment approach. At the time of writing, the values being used in most assessments are still being discussed and revised.

The three collections selected are: fish, minerals and vascular plants. These represent the three major groups of natural science collections: zoological, geological and botanical. Full documentation on most of the risk assessment results and on details of the methodology will be published in the discipline-specific or collection care literature over the next few years.

The magnitudes of risk are shown in the form of histograms in Figure 4. Risks which have an estimated magnitude of less than $0.0001(0.01 \%)$ loss in value to the collection over 100 years are not shown. for the sake of clarity. This does not mean that such risks were ignored completely. Where they could be mitigated by a simple, low-cost change in hardware or procedure, this was implemented immediately. If the risk affected only a small fraction of the collection, that fact was noted. Ideally, the value of the fraction of the collection affected, as a part of the total value of the collection, would have been incorporated into the loss in value term in the calculation of magnitude of risk. Still, some judgment is required. In cases where that fraction of the collection is considered too important to accept the level of risk, then the risk will be given special consideration.

Overall, most collections were at significant risk $(>0.0001)$ from less than half of the 23 identified categories of risk. The particular categories of risk most affecting each collection varied depending on the nature of the collection.

3.2 Fish collection

The fish collection comprises approximately one million specimens stored in 100,000 jars. For this collection the greatest risk identified was the equivalent for a fluid-preserved collection of an 'incorrect relative humidity type 3 ' risk, that is, deterioration from storage at too low concentration of alcohol. This high level of risk results from a large number of specimens being held in small containers that are not well sealed. Preferential evaporation of alcohol from the preservative solution results in low preservative concentrations. Finding improved methods of sealing containers has been given a high priority.

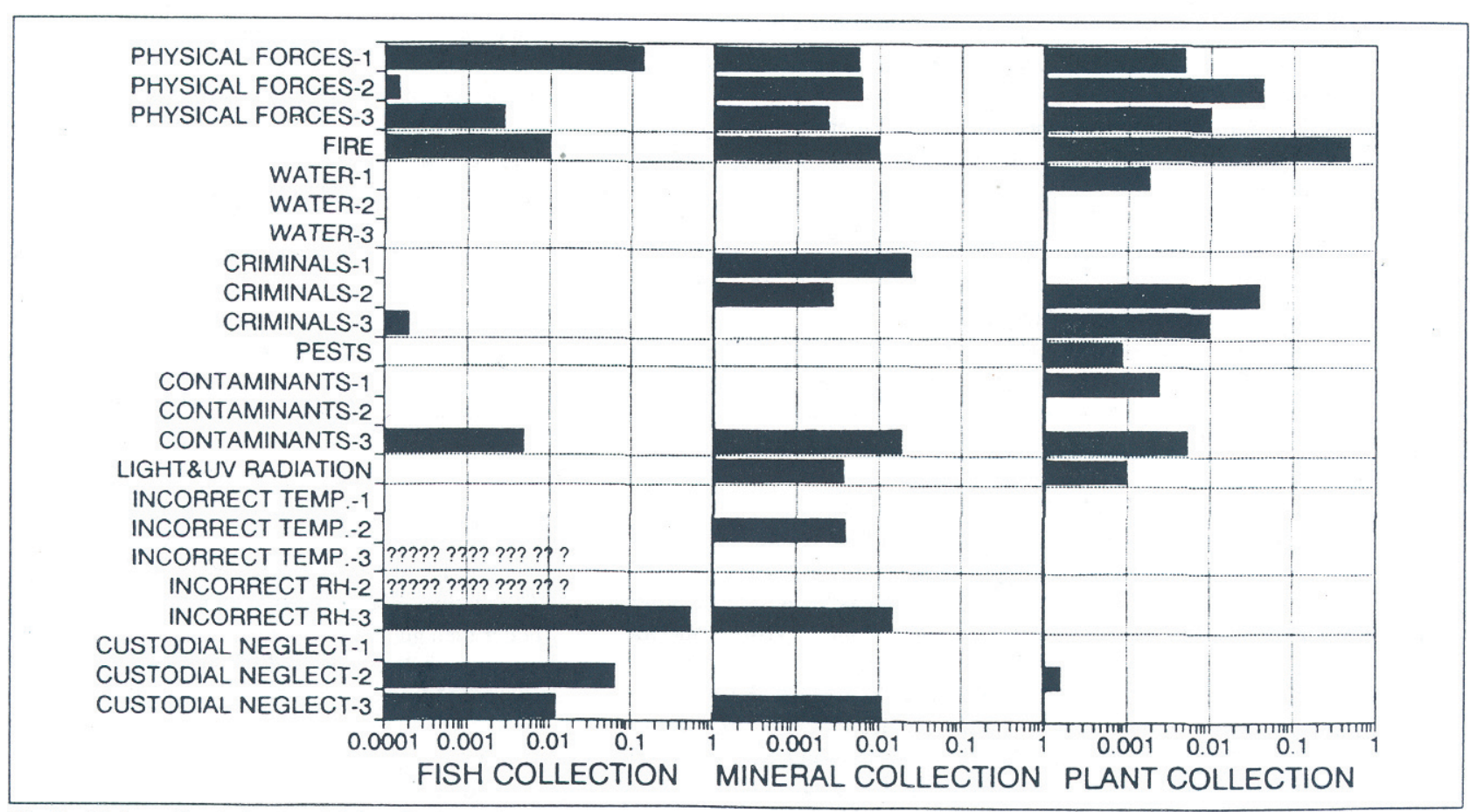

Fig. 4 Estimated magnitudes of risks affecting fish, mineral and plant collections. Risks are expressed in terms of anticipated loss in value, as a fraction of total value, to the existing collection over a 100 -year period, assuming current conditions persist. 
The next most serious risk is in the category 'physical forces type I'. This is the risk of jars of fluid-preserved specimens falling off shelves during a moderate earthquake. The third highest risk is in the category of 'custodial neglect type 2'. This risk primarily involves loss of material acquired at a much faster rate than it can be processed. The material is now receiving minimal care while higher priority issues are addressed. The magnitude of risk could be reduced to reflect the lower current value for research of this unprocessed material. Risks in the categories of 'incorrect temperature type 3' and 'incorrect relative humidity type 2 ' relate to storing the collection at a higher than optimal temperature and damage to specimens from osmotic shock due to too rapid a change in preservative concentration, respectively. Inadequate knowledge of the significance of these factors prevents even an educated guess at the magnitudes of these risks.

\subsection{Plant collection}

The plant collection contains approximately 600,000 specimens mounted on herbarium sheets. Fire appears to be the greatest risk to this collection. It is housed in a leased, five-storey building, shared with non-museum tenants and having no automatic fire suppression system. The estimated level of risk due to fire does not appear unreasonable but may be significantly revised once fire control experts have reviewed the situation and the estimated probability. The second greatest risk is of physical damage during handling. The third most significant risk is due to criminals and is primarily associated with unreturned loans. It was surprising that these risks were determined to be significantly higher than the risk due to pests. This results from the facts that the collection is extensively used and that an effective pest management program is in place.

\subsection{Mineral collection}

The mineral collection contains approximately 36,000 catalogued specimens. For this collection, the magnitudes of the 10 highest risks are all within one order of magnitude, ranging from 0.025 to 0.0028 . Since the accuracy is thought to be no better than one order of magnitude, each of these 10 risks must, at this time, be considered approximately equal. One noteworthy result was that obtained for the category incorrect temperature type 2'. This category relates to damage due to thermal shock, induced by exhibit lighting or by transfer into and between wash and rinse solutions of unequal temperature. The latter contributed to the high magnitude of risk. Two factors make this a high risk. First, the fraction of the collection that is most susceptible correlates closely with the fraction of the collection that has the highest display value and second, a single instance results in a high loss in value. Although the extent to which this occurs is estimated to be twice per year, the magnitude of risk over the 100 -year period was calculated as 0.004 . This is an example of a risk that can be mitigated simply by ensuring that staff preparing specimens are aware of its significance and take adequate precautions during preparation treatments.

\section{COST, BENEFITS AND DIFFICULTIES}

Staff time was the major investment in performing this assessment. To date, approximately three person-years have been used. It is estimated that a total of four-person years will be required before all assessments are through their final revisions and the conclusions needed for setting collection care priorities are extracted. This represents approximately $10 \%$ of the time and salary budget annually allocated to collection care at the Canadian Museum of Nature. The project cost might be reduced by half if the project were repeated. This saving would result from an ability to express more clearly to the staff our expectations of what is required. For other museums, the cost would be similar for institutions of similar size. The cost, as a proportion of total collection care costs, would probably be greater for smaller institutions.

The exercise of performing the risk assessment, even aside from the results, produces many benefits. Information necessary for management decisions on priorities for addressing collection care problems is available for all collections in a clear and consistent form. Knowledge of the expected rate of occurrence of problems, that often resides only in the memories of long-term staff members, can be effectively recorded. A sense of the value of their own ideas and knowledge is instilled in staff. Collection care staff tend to accept changes because they were intimately involved in defining problems and proposing and evaluating possible solutions.

Staff learn through discovery. When they see that a seemingly unimportant pan of their job makes a large difference in risks to collections, they are more likely to alter their work habits appropriately than if they are simply told to change. A team spirit is fostered which reduces the chance of collection care staff becoming resentful of funding priorities being set on collections other than those with which they most associate. Guidance is provided in the area of conservation research priorities.

Although there are numerous benefits, there are also many difficulties in doing a risk assessment. Many kinds of damage to collections are rate-dependent on several parameters. As one example, the rate of disintegration of plant specimen labels written on acidic, lignin-rich papers is determined by contaminant concentration, relative humidity, temperature and physical forces. Further, since the result of this disintegration is loss of specimen data, the risk could be considered a product of custodial neglect. In order to ensure that risks are not counted twice, this damage was considered to arise from a single category of risk, in this case, 'contaminant type 3'. The category of risk selected as responsible for the damage was chosen to reflect the parameter which can most easily be controlled to limit the rate of damage. It is important to remember that several parameters can be controlled simultaneously to obtain the most cost-effective risk reduction.

For many categories of risk, we do not have an adequate understanding of the deterioration processes, and their effect on the value of specimens, to make a reasonable estimate of the magnitude of risk. Examples include the long-term effect of low-level contaminant concentrations on the research value of skeletal material or fluid-preserved specimens. This challenges conservation researchers to focus and interpret their work in a way that will relate their results to overall collection care requirements. Experts in the assessment of 'type 1' risks are already achieving this [8-10].

Estimation of loss in value is a difficult undertaking. At present, it can only be a very subjective estimate based on the collection manager's knowledge of the use of damaged specimens for various purposes. Improving the ability to estimate loss in value will require effective communication between conservators, collection managers, and researchers and other users of collections over many years.

Finally, it is difficult to keep the uncertainties of the estimated magnitudes of risks in perspective while interpreting the results. The quality of the estimates varies from highly accurate to little better than guesses. When the assessment project is repeated, two estimates will be obtained for each category of risk, to reflect the upper and lower boundaries of uncertainty.

\section{CONCLUSION}

It is possible to categorize all risks to collections in a way that enables a museum to consider total risk to its collections. At 
present, inadequate knowledge of many factors that determine the magnitude of risks severely limits the ability to quantify many of the risks. Nevertheless, the process of having all collection care staff work together to attempt to produce a complete risk assessment for the collections of an institution provides benefits that justify the effort.

From an institutional perspective, the worth of the risk assessment, beyond the benefits derived from performing the exercise, can only be determined as it is used to set collection care priorities over the years. From a broader perspective, striving to understand the relative significance of all risks to collections is seen as an essential endeavour. Only when this can be done will the ideal situation be achieved where practitioners of preventive conservation will be able to quantify all risks to a set of collections and determine the most cost-effective means of reducing overall risk to those collections.

\section{ACKNOWLEDGEMENTS}

The author is grateful to his many colleagues who have offered encouragement and insightful commentary over the years that this method was developed, especially Stefan Michalski, Paul Marcon and Charlie Costain of the Environment and Deterioration Research Division, Canadian Conservation Institute. Sylvie Marcil, Canadian Museum of Nature. worked to clarify and refine concepts and definitions in order to ensure consistency of interpretation among collection management staff. The author is most grateful to the staff of the Canadian Museum of Nature, Collections Division, who worked hard, patiently and diligently to apply this method to the collections in their care.

\section{REFERENCES}

1 Michalski. S., 'An overall framework for preventive conservation and remedial conservation' in ICOM Committee for Conservation 9th Triennial Meeting, Dresden (1990) 589-591.
2 Babcock. P., 'Ready for the worst' Museum News. May/June (1990) 50-54.

3 Roberts. B.O., 'Establishing a disaster prevention/response plan, an international perspective and assessment'. Technology and Conservation 4 (1993) 15-17, 35-36.

4 Waller. R., and Shelton. S., 'Risk management strategies applied to the development of pragmatic solutions to collections storage probems' in Programme and Abstracts of the Fourth Annual Meeting of the Society for the Preservation of Natural History, Collections. Calgary (1989) 32.

5 Baer, N.S., 'Assessment and management of risks to cultural property' in Science. Technology and European Cultural Heritage. Butterworth-Heinemann. Oxford (1991) 27-36.

6 Moore. P.G., The Business of Risk. Cambridge University Press (1983).

7 Crouch. E.A.C., and Wilson. R., Risk/Benefit Analysis. Ballinger Publishing Company. Cambridge MA (1982).

8 Earthquake Risk Assessment, Swiss Reinsurance Company. Zurich (1982).

9 Agbabian, M.S., Ginell, W.S., Masri. S.F. and Nigbor. R.L. 'Evaluation of earthquake damage mitigation method for museum objects'. Studies in Conservation 36 (1991) 111-120.

10 Harmathy, T.Z., et al., 'A decision logic for trading between fire safety measures', Fire and Materials 14 (1989) I-10.

AUTHOR

Robert Waller received a BSc from the University of Manitoba. After working for 15 years on conservation of mineral specimens and collections he became Head of Conservation, then Chief of Collections at The Canadian Museum of Nature. He is currently also Chair of the Canadian Association of Professional Conservators (CAPAC). Address: Collections Division, Canadian Museum of Nature, Box 3443. Station 'D', Ottawa. Ontario, Canada KIP 6P4. 